\title{
TOPOLOGY OPTIMIZATION OF COMPLIANT MECHANISMS USING THE HOMOGENIZATION METHOD
}

\author{
SHINJI NISHIWAKI*, MARY I. FRECKER, SEUNGJAE MIN AND NOBORU KIKUCHI
}

Department of Mechanical Engineering and Applied Mechanics, University of Michigan, Ann Arbor MI 48109-2125, U.S.A.

\begin{abstract}
A procedure to obtain a topology of an optimal structure considering flexibility is presented. The methodology is based on a mutual energy concept for formulation of flexibility and the homogenization method. A multi-objective optimization problem is formulated as an application of compliant mechanism design. Some examples of the design of compliant mechanisms for plane structures are presented. (C) 1998 John Wiley \& Sons, Ltd.
\end{abstract}

KEY WORDS: optimal design; topology optimization; compliant mechanisms; homogenization method; mutual energy

\section{INTRODUCTION}

In general, the stiffest structure has been considered optimal. Most structural optimization problems are formulated by minimizing the compliance of a structure as an objective function. However, it is possible that better performance can be obtained with a flexible structure rather than the stiffest structure if flexibility is efficiently implemented in the structure. Moreover, flexible parts can offer mechanical function to the structure. An example of structure with mechanical function is a compliant mechanism. Compliant mechanisms are a relatively new breed of jointless mechanisms which utilize elastic deformation as source of motion. They are designed to be intentionally flexible, and this flexibility allows the structure to function as a mechanism. As noted by Midha et al., ${ }^{1}$ compliant mechanisms are desirable since they require fewer parts, and have less wear, noise, and backlash than their rigid-body counterparts. Hence, the design of compliant mechanisms is an example of how flexible structures can provide better performance than stiff structures.

There are two approaches for compliant mechanism design, a kinematic synthesis approach and a continuum synthesis approach, as explained by Frecker et al. ${ }^{2}$ Midha $^{1}$ and his associates were pioneers in developing the kinematic approach for compliant mechanism synthesis. This method is based on traditional rigid-body kinematics. The basic configuration of the mechanism

\footnotetext{
* Correspondence to: Shinji Nishiwaki, Department of Mechanical Engineering and Applied Mechanics, University of Michigan, 3005 EECS, Ann Arbor, MI 48109-2125, U.S.A. E-mail: shinji@engin.umich.edu
}

Contract/grant sponsor: National Science Foundation; Contract/grant number: DMI 9622261

Contract/grant sponsor: TOYOTA Central R\&D Labs, Inc.

CCC 0029-5981/98/030535-25\$17.50

(C) 1998 John Wiley \& Sons, Ltd. 
design is obtained by knowledge from kinematics, and is converted to a partially compliant mechanism with flexural segments or a fully compliant mechanism with lumped compliance. For example, Her and Midha $^{3}$ discussed kinematic properties of compliant mechanisms such as degree of compliance using this rigid body mechanism analysis, and presented a methodology for deriving all possible compliant mechanisms from a given rigid-body kinematic chain. Murphy et al. $^{4}$ extended a topological synthesis approach for rigid-body mechanisms to compliant mechanism synthesis using graph theory. This approach determines the number of flexural segments, connection type and kinematic inversions. Howell and Midha ${ }^{5}$ developed a pseudo-rigid-body model to aid in the design of compliant mechanisms composed of small flexible pivots and relatively rigid links, and constructed a design method ${ }^{6}$ using this model. Moreover, they improved this model in order to deal with large deflections. ${ }^{7}$ These procedures, however, are limited to compliant mechanisms with lumped compliance such as flexible pivots.

The second major approach for the design of compliant mechanisms is a continuum synthesis for the design of distributed compliant mechanisms. This method is based on topology optimization methods for structures. Sigmund ${ }^{8}$ proposed a modified approach using microstructure design of materials based on optimal configuration of an elastic truss. He presented the compliant mechanism design by solving an optimization problem in which the objective function is to minimize the total volume with prescribed output motions. Larsen et al. ${ }^{9}$ developed another approach based on microstructure design. In this method, the mechanism design is obtained by solving a different optimization problem in which the objective function is to minimize the error in obtaining prescribed values of the geometrical and mechanical advantages. However, it is difficult to design compliant mechanisms that provide the flexibility required for kinematic function and the rigidity required for structural function simultaneously, since the output constraint must be specified beforehand.

Another topology optimization approach is based on the homogenization method. This method was proposed by Bendsøe and Kikuchi. ${ }^{10}$ Prior to this, Cheng and Olhoff ${ }^{11}$ reported important characteristics about the optimal design of solid elastic plates. They pointed out that a global optimal solution generally does not exist within the class of smooth functions, or within the class of smooth functions with a finite number of discontinuities. This result led to a series of works on optimal design problems using a microstructure. Kohn and Strang ${ }^{12}$ introduced a 'relaxation' concept to deal with an ill-posed variational problem that can be formulated for optimal design. This concept implies that the introduction of microstructure and use of the homogenization method allow an optimal solution. Rozvany et al. ${ }^{13}$ investigated the implication of this relaxation concept with the design of perforated elastic plates. Bendsøe and Kikuchi ${ }^{10}$ constructed a methodology for topology optimization using the homogenization method. This new optimization approach created a paradigm shift in the structural optimization field. Suzuki and Kikuchi ${ }^{14}$ established a static optimization problem in which the objective is to minimize the mean compliance. This approach was also extended to dynamic problems. Díaz and Kikuchi ${ }^{15}$ proposed an eigenvalue optimization method in which the objective function is to maximize a single eigenvalue. Ma et al. ${ }^{16}$ constructed a multi-eigenvalue optimization method with the convex approach in the optimization process. They also extended this approach to a frequency response optimization problem. ${ }^{17}$ Ananthasuresh ${ }^{18}$ extended this approach to the design of compliant mechanisms at first. However, his results seem to be rather the maximum stiffness design than the mechanism design. That is, the optimal structures do not have sufficient flexibility as the compliant mechanism because of the formulation of the multi-objective optimization 
problem. Thus, this optimization method for the design of flexible structures like compliant mechanisms has not been established using the homogenization method.

This paper describes a topology optimization method considering flexibility using the homogenization method. This method is applied to the concept to the design of compliant mechanisms. First, mutual mean compliance is introduced in order to define the flexibility using a mutual energy concept. The sensitivity of the mutual mean compliance with respect to the design variables required in optimization procedure is formulated with variational calculus. Formulation of the rigidity is also derived in a way similar to the derivation of the flexibility. Next, a new multi-objective problem is constructed for the design of compliant mechanisms, along with verification of the characteristics of the multi-criteria objective function. Three examples are presented in order to examine the configuration of optimal solutions. These confirm that the concept of flexible structure design can be used to design compliant mechanisms.

\section{FORMULATION OF MUTUAL MEAN COMPLIANCE AND SENSITIVITY ANALYSIS}

Mutual mean compliance is introduced to formulate the flexibility of a structure using a mutual energy concept. ${ }^{19,20}$ Consider a linear elastic body occupying a two-dimensional domain, $\Omega$, as shown in Figure 1. Suppose that the body is subjected to boundary traction $\mathbf{t}^{1}$ at boundary $\Gamma t^{1}$ in case (a), and boundary traction $\mathbf{t}^{2}$ at boundary $\Gamma t^{2}$ in case (b). Body forces applied to the elastic body are ignored for simplicity in the formulation. The displacement field is $\mathbf{u}^{1}=\left\{u_{1}^{1}, u_{2}^{1}\right\}$ in case (a), and $\mathbf{u}^{2}=\left\{u_{1}^{2}, u_{2}^{2}\right\}$ in case (b). The mutual mean compliances of the structures, $L^{1}\left(\mathbf{u}^{2}\right)$ and $L^{2}\left(\mathbf{u}^{1}\right)$, are defined by the following load linear forms:

$$
\begin{array}{ll}
L^{1}\left(\mathbf{u}^{2}\right)=\int_{\Gamma t^{1}} \mathbf{t}^{1} \cdot \mathbf{u}^{2} \mathrm{~d} \Gamma=\int_{\Gamma t^{1}} t_{i}^{1} u_{i}^{2} \mathrm{~d} \Gamma \quad \mathbf{u}^{2} \in V \\
L^{2}\left(\mathbf{u}^{1}\right)=\int_{\Gamma t^{2}} \mathbf{t}^{2} \cdot \mathbf{u}^{1} \mathrm{~d} \Gamma=\int_{\Gamma t^{2}} t_{i}^{2} u_{i}^{1} \mathrm{~d} \Gamma \quad \mathbf{u}^{1} \in V
\end{array}
$$

where $V$ is the admissible linear space such that

$$
V=\left\{\mathbf{v}=v_{i} \mathbf{e}_{i}: v_{i} \in H^{1}(\Omega) \text { with } \mathbf{v}=0 \text { on } \Gamma_{d} i=1,2\right\}
$$
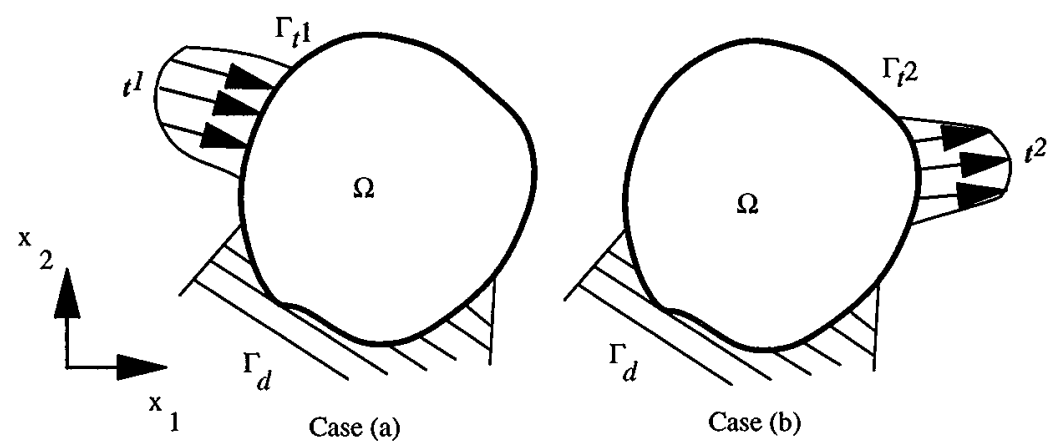

Figure 1. An elastic body subjected to two different tractions 
Assuming that boundary traction $\mathbf{t}^{1}$ in case (a) is an applied force, and boundary traction $\mathbf{t}^{2}$ in case (b) is a unit dummy load, equation (2) shows the measurement of deformation at $\Gamma t^{2}$ along a specified direction defined by $\mathbf{t}^{2}$ when $\mathbf{t}^{1}$ is applied at $\Gamma t^{1}$. Here, the flexibility is defined by how much the elastic body deforms at $\Gamma t^{2}$ when $\mathbf{t}^{1}$ is applied at $\Gamma t^{1}$. The formulation of the mutual mean compliance is interpreted as the measurement of flexibility between $\Gamma t^{1}$ and $\Gamma t^{2}$. Hence, sufficient flexibility can be obtained by maximizing the mutual mean compliance. The bilinear form is introduced as

$$
a(\mathbf{u}, \mathbf{v})=\int_{\Omega} \boldsymbol{\varepsilon}(\mathbf{v})^{\mathrm{T}} \mathbf{E} \boldsymbol{\varepsilon}(\mathbf{u}) \mathrm{d} \Omega=\int_{\Omega} E_{i j k l} \varepsilon_{k l}(\mathbf{u}) \varepsilon_{i j}(\mathbf{v}) \mathrm{d} \Omega
$$

with linearized strains

$$
\varepsilon_{i j}(\mathbf{u})=\frac{1}{2}\left(\frac{\partial u_{i}}{\partial x_{j}}+\frac{\partial u_{j}}{\partial x_{i}}\right)
$$

where $E_{i j k l}$ is the elasticity tensor of the linear elastic body.

Each displacement field satisfies the principle of virtual work in the sense of mutual energy,

$$
\begin{array}{lll}
a\left(\mathbf{u}^{1}, \mathbf{v}^{2}\right)=L^{1}\left(\mathbf{v}^{2}\right) & \text { for } \mathbf{u}^{1} \in V & \forall \mathbf{v}^{2} \in V \\
a\left(\mathbf{u}^{2}, \mathbf{v}^{1}\right)=L^{2}\left(\mathbf{v}^{1}\right) & \text { for } \mathbf{u}^{2} \in V & \forall \mathbf{v}^{1} \in V
\end{array}
$$

The total potential energy in the sense of mutual energy is defined by

$$
F\left(\mathbf{v}^{1}, \mathbf{v}^{2}\right)=\frac{1}{2} a\left(\mathbf{v}^{1}, \mathbf{v}^{2}\right)-\frac{1}{2} L^{1}\left(\mathbf{v}^{2}\right)-\frac{1}{2} L^{2}\left(\mathbf{v}^{1}\right)
$$

Since the following relation is obtained at equilibrium:

$$
L^{2}\left(\mathbf{u}^{1}\right)=a\left(\mathbf{u}^{2}, \mathbf{u}^{1}\right)=a\left(\mathbf{u}^{1}, \mathbf{u}^{2}\right)=L^{1}\left(\mathbf{u}^{2}\right)
$$

the total potential energy satisfies at equilibrium,

$$
F\left(\mathbf{u}^{1}, \mathbf{u}^{2}\right)=\frac{1}{2} a\left(\mathbf{u}^{1}, \mathbf{u}^{2}\right)-\frac{1}{2} L^{1}\left(\mathbf{u}^{2}\right)-\frac{1}{2} L^{2}\left(\mathbf{u}^{1}\right)=-\frac{1}{2} L^{2}\left(\mathbf{u}^{1}\right)
$$

Let $A$ be the design variable, and consider $F$ a function of $\mathbf{u}^{1}, \mathbf{u}^{2}$, and $A$. Taking the first variation of $F$ with respect to $\mathbf{u}^{1}, \mathbf{u}^{2}$, and $A$ at equilibrium:

$$
\delta F\left(\mathbf{u}^{1}, \mathbf{u}^{2}, A\right)=D F\left(\mathbf{u}^{1}\right)\left(\delta \mathbf{u}^{1}\right)+D F\left(\mathbf{u}^{2}\right)\left(\delta \mathbf{u}^{2}\right)+D F(A)(\delta A)
$$

where $D F(\mathbf{u})(\mathbf{v})$ is the G-differential such that

$$
D F(\mathbf{u})(\mathbf{v})=\lim _{\xi \rightarrow 0} \frac{\partial}{\partial \xi} F(\mathbf{u}+\xi \mathbf{v})
$$

The first variation of $F$ yields

$$
\begin{aligned}
\delta F\left(\mathbf{u}^{1}, \mathbf{u}^{2}, A\right)= & \frac{1}{2} a\left(\mathbf{u}^{1}, \frac{\partial \mathbf{u}^{2}}{\partial A} \delta A+\delta \mathbf{u}^{2}\right)-\frac{1}{2} L^{1}\left(\frac{\partial \mathbf{u}^{2}}{\partial A} \delta A+\delta \mathbf{u}^{2}\right)+\frac{1}{2} a\left(\mathbf{u}^{2}, \frac{\partial \mathbf{u}^{1}}{\partial A} \delta A+\delta \mathbf{u}^{1}\right) \\
& -\frac{1}{2} L^{2}\left(\frac{\partial \mathbf{u}^{1}}{\partial A} \delta A+\delta \mathbf{u}^{1}\right)+\frac{1}{2} \int_{\Omega} \boldsymbol{\varepsilon}\left(\mathbf{u}^{2}\right)^{\mathrm{T}} \frac{\partial \mathbf{E}}{\partial A} \boldsymbol{\varepsilon}\left(\mathbf{u}^{1}\right) \mathrm{d} \Omega \delta A
\end{aligned}
$$


Since $\mathbf{v}^{2}$ in equation (5) and $\mathbf{v}^{1}$ in equation (6) are arbitrary variables, the first four terms in equation (11) cancel by setting $\mathbf{v}^{2}=\left(\partial \mathbf{u}^{2} / \partial A\right) \partial A+\delta \mathbf{u}^{2}$ and $\mathbf{v}^{1}=\left(\partial \mathbf{u}^{1} / \partial A\right) \delta A+\delta \mathbf{u}^{1}$. Therefore, the sensitivity of $F$ with respect to $A$ is given by

$$
\frac{\partial F}{\partial A}=\frac{1}{2} \int_{\Omega} \boldsymbol{\varepsilon}\left(\mathbf{u}^{2}\right)^{\mathrm{T}} \frac{\partial \mathbf{E}}{\partial A} \boldsymbol{\varepsilon}\left(\mathbf{u}^{1}\right) \mathrm{d} \Omega
$$

Using equation (8), the sensitivity of the mutual mean compliance $L^{2}\left(\mathbf{u}^{1}\right)$ is obtained as follows:

$$
\frac{\partial L^{2}\left(\mathbf{u}^{1}\right)}{\partial A}=-\int_{\Omega} \boldsymbol{\varepsilon}\left(\mathbf{u}^{2}\right)^{\mathrm{T}} \frac{\partial \mathbf{E}}{\partial A} \boldsymbol{\varepsilon}\left(\mathbf{u}^{1}\right) \mathrm{d} \Omega
$$

We will now consider the ordinary mean compliance. If only case (a) (in Figure 1) is considered, ordinary mean compliance is also expressed as

$$
L^{1}\left(\mathbf{u}^{1}\right)=\int_{\Gamma t^{1}} \mathbf{t}^{1} \cdot \mathbf{u}^{1} \mathrm{~d} \Gamma=\int_{\Gamma t^{1}} t_{i}^{1} u_{i}^{1} \mathrm{~d} \Gamma \quad \mathbf{u}^{1} \in V
$$

Here, the rigidity is defined by how little the elastic body deforms at $\Gamma t^{1}$ when $t^{1}$ is applied at $\Gamma t^{1}$. The formulation of the ordinary mean compliance is interpreted as the measurement of the rigidity at $\Gamma t^{1}$. Hence, sufficient rigidity can be obtained by minimizing the mean compliance. Following the same procedure as above, the sensitivity of $L^{1}\left(\mathbf{u}^{1}\right)$ with respect to $A$ is obtained by

$$
\frac{\partial L^{1}\left(\mathbf{u}^{1}\right)}{\partial A}=-\int_{\Omega} \boldsymbol{\varepsilon}\left(\mathbf{u}^{1}\right)^{\mathrm{T}} \frac{\partial \mathbf{E}}{\partial A} \boldsymbol{\varepsilon}\left(\mathbf{u}^{1}\right) \mathrm{d} \Omega
$$

This formulation is identical to the sensitivity of the mean compliance which Suzuki and Kikuchi $^{14}$ introduced when constructing the minimal mean compliance design problem.

\section{HOMOGENIZATION METHOD}

Topology optimization using the homogenization method is briefly discussed in this section. The original idea was introduced by Bendsøe and Kikuchi. ${ }^{10}$ The problem is posed as optimizing the material distribution in a perforated structure with infinite microscale voids. In the optimization process, the portions of the perforated structure that are filled with the material can be identified as a solid structure. On the other hand, the portions that are filled with voids can be identified as no structure. Using the homogenization method, the design domain can be relaxed to deal with the nonsmooth characteristics of optimal configurations like the perforated structure that Kohn and Strang ${ }^{12}$ discussed.

Consider the unit cell of a microstructure shown in Figure 2. For simplicity, a two- dimensional problem is considered. It is assumed that this microstructure is formed inside an empty rectangle in a unit cell, where $\alpha, \beta$, and $\theta$ are regarded as the design variables. In order to develop a complete void, both $\alpha$ and $\beta$ should be 1 , whereas $\alpha$ and $\beta$ should be 0 for solid material. The variable $\theta$ represents the rotation of the unit cell.

The elasticity tensor of perforated structures, $\mathbf{E}^{\varepsilon}$, is given by equation (16). The parameter $\varepsilon$ represents the periodicity and is assumed to be small:

$$
\mathbf{E}^{\varepsilon}(\mathbf{x})=\mathbf{E}(\mathbf{x}, \mathbf{y}), \quad \mathbf{y}=\mathbf{x} / \varepsilon
$$




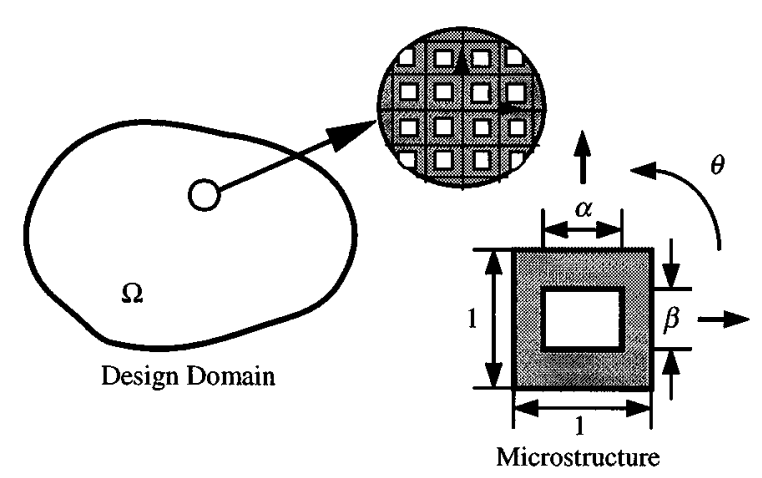

Figure 2. A unit cell of a microstructure

The homogenized elasticity tensor, $\mathbf{E}^{\mathrm{H}}$, can be computed by solving the characteristic deformations $\chi$ satisfying the following equation:

$$
\int_{Y} \varepsilon_{y}(\mathbf{v})^{\mathrm{T}} \mathbf{E}(\mathbf{x}, \mathbf{y}) \varepsilon_{y}(\chi(\mathbf{x}, \mathbf{y})) \mathrm{d} Y=\int_{Y} \varepsilon_{y}(\mathbf{v})^{\mathrm{T}} \mathbf{E}(\mathbf{x}, \mathbf{y}) \mathrm{d} Y \quad \forall \mathbf{v} \in V_{y}
$$

where

$$
\boldsymbol{\varepsilon}_{y}()=\left\{\frac{\partial}{\partial y_{1}} \frac{\partial}{\partial y_{2}} \frac{\partial}{\partial y_{2}}+\frac{\partial}{\partial y_{1}}\right\}
$$

$V_{y}$ is the admissible space defined in the cell $Y$,

$$
V_{y}=\left\{\mathbf{v}=v_{i} \mathbf{e}_{i}: v_{i} \in H^{1}(Y) \mid \mathbf{v} \text { is } Y \text { - periodic in the unit cell } Y\right\}
$$

After obtaining characteristic deformations, $\chi$, the homogenized elasticity tensor, $\mathbf{E}^{\mathrm{H}}$, with respect to the principle coordinate axes of the material, is computed by

$$
\mathbf{E}^{\mathrm{H}}=\frac{1}{|\mathbf{Y}|} \int_{\mathbf{Y}} \mathbf{E}(\mathbf{x}, \mathbf{y})\left(\mathbf{I}-\varepsilon_{y}(\chi)\right) \mathrm{d} Y
$$

where $|\mathbf{Y}|$ stands for the area of $Y$.

Furthermore, when the unit cell is rotated by the angle $\theta$ as shown in Figure 2, the homogenized elasticity tensor, $\mathbf{E}^{\mathrm{G}}$, is computed by the following equation:

$$
\mathbf{E}^{\mathrm{G}}=\mathbf{R}(\theta)^{\mathrm{T}} \mathbf{E}^{\mathrm{H}} \mathbf{R}(\theta),
$$

where $\mathbf{R}$ is the rotation matrix.

One can see that $\mathbf{E}^{\mathbf{G}}$ is a function of the microscopic design variables $\alpha, \beta$, and $\theta$, and that the optimization problem can be posed in terms of these microscopic variables using $\mathbf{E}^{\mathrm{G}}$.

\section{MULTI-OBJECTIVE OPTIMIZATION FOR COMPLIANT MECHANISM DESIGN}

Compliant mechanism design requires two different functions, a kinematic function and a structural function, as shown in Figure 3. The compliant mechanism must have a kinematic function, 


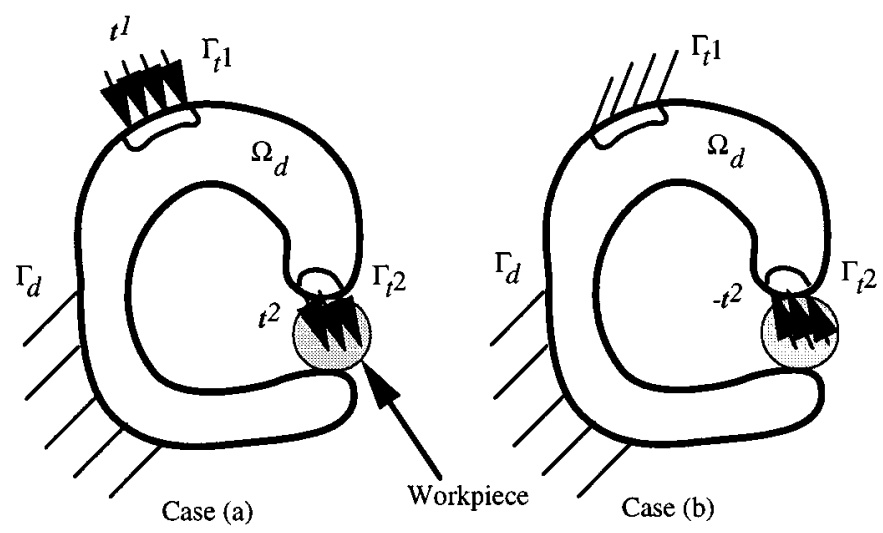

Figure 3. Compliant mechanism

or motion, which is provided by the sufficient flexibility between $\Gamma t^{1}$ and $\Gamma t^{2}$. That is, the compliant mechanism must deform along a direction specified by a dummy load $\mathbf{t}^{2}$ at specified region $\Gamma t^{2}$ (case (a)). Here, the mechanism is subjected to an applied traction $\mathbf{t}^{1}$ at $\Gamma t^{1}$. It is noted that the compliant mechanism may deform considerably in its use. However, it is possible to ignore the effect of large deformation in the topology design phase, if our goal in this design phase is that the compliant mechanism must qualitatively deform in the desired direction of motion. In other words, the small deformation/rotation assumption is appropriate only if we take into account the qualitative characteristics of the mechanism function. Conversely, if the quantitative performance of the deformation in the designed mechanism is the purpose of design optimization, we must include large deformation analysis in compliant mechanism design. Since our goal is to seek the optimum design that qualitatively provides the initial motion of the mechanism in the specified direction, the topology of the compliant mechanism can be determined under the small deformation/rotation assumption. However, it is also noted that the designed configuration must be subsequently examined to determine whether it generates the motion that we originally intended after the topology design phase, since the design here shall be determined in the context of small deformation linear elasticity. In the following section, the designed configuration will be examined using the large displacement finite element analysis. Based on this argument, the kinematic function can be achieved by maximizing the mutual mean compliance between $\Gamma t^{1}$ and $\Gamma t^{2}$.

The compliant mechanism must also have a structural function which is provided by sufficient rigidity at $\Gamma t^{2}$ in order to resist reaction forces posed by a workpiece (case (b)). That is, after the compliant mechanism contacts the workpiece, it must be stiff enough to resist the reaction force that is exerted by the workpiece once it has been secured. This rigidity allows the compliant mechanism to maintain its shape to hold the workpiece. This structural function is obtained by minimizing the mean compliance posed by the traction $\mathbf{t}^{2}$ while $\Gamma t^{1}$ is fixed, since the compliant mechanism should maintain the deflection while it is subjected to traction $\mathbf{t}^{1}$ at $\Gamma t^{1}$.

The kinematic function can be obtained by solving the following optimization problem:

$$
\underset{\alpha, \beta, \text { and } \theta}{\operatorname{maximize}} L^{2}\left(\mathbf{u}^{1}\right)=\int_{\Gamma t^{2}} \mathbf{t}^{2} \cdot \mathbf{u}^{1} \mathrm{~d} \Gamma
$$




$$
\begin{aligned}
& \text { subject to } a\left(\mathbf{u}^{1}, \mathbf{v}^{1}\right)=L^{1}\left(\mathbf{v}^{1}\right) \text { for } \mathbf{u}^{1} \in V^{(a)} \forall \mathbf{v}^{1} \in V^{(a)} \\
& a\left(\mathbf{u}^{2}, \mathbf{v}^{2}\right)=L^{2}\left(\mathbf{v}^{2}\right) \quad \text { for } \mathbf{u}^{2} \in V^{(a)} \forall \mathbf{v}^{2} \in V^{(a)} \\
& 0 \leqslant \alpha \leqslant \alpha_{\text {upp }}<1 \\
& 0 \leqslant \beta \leqslant \beta_{\text {upp }}<1 \\
& g(\alpha, \beta)=\int_{\Omega_{\mathrm{d}}}(1-\alpha \beta) \mathrm{d} \Omega-\Omega_{\mathrm{s}} \leqslant 0
\end{aligned}
$$

where $\mathbf{V}^{(a)}=\left\{\mathbf{v}=v_{i} \mathbf{e}_{i}: v_{i} \in H^{1}(\Omega)\right.$ with $v=0$ on $\left.\Gamma_{\mathrm{d}}\right\}, \alpha_{\text {upp }}$ and $\beta_{\text {upp }}$ are the upper bounds of the microscopic variables, $\alpha$ and $\beta$, respectively. These are specified in order to avoid the singularity in the structural analysis. $\Omega_{\mathrm{d}}$ is the design domain of the compliant mechanism, and $\Omega_{\mathrm{s}}$ is the total volume constraint of the solid material forming the porous structure. Note that equation(20c) is required in order to obtain the sensitivity of the objective function in the optimization process even though $\mathbf{u}^{2}$ does not appear explicitly in the formulation of the optimization problem. This implies that this optimization problem requires equation (20c) as a constraint.

The structural function can be obtained by solving the following optimization problem:

$$
\begin{aligned}
\underset{\alpha, \beta, \text { and } \theta}{\operatorname{minimize}} L^{3}\left(\mathbf{u}^{3}\right) & =\int_{\Gamma t^{2}} \mathbf{t}^{3} \cdot \mathbf{u}^{3} \mathrm{~d} \Gamma \\
\text { subject to } \mathbf{t}^{3} & =-\mathbf{t}^{2} \\
a\left(\mathbf{u}^{3}, \mathbf{v}^{3}\right) & =L^{3}\left(\mathbf{v}^{3}\right) \quad \text { for } \mathbf{u}^{3} \in V^{(b)} \forall \mathbf{v}^{3} \in V^{(b)} \\
0 & \leqslant \alpha \leqslant \alpha_{\text {upp }}<1 \\
0 & \leqslant \beta \leqslant \beta_{\text {upp }}<1 \\
g(\alpha, \beta) & =\int_{\Omega_{\mathrm{d}}}(1-\alpha \beta) \mathrm{d} \Omega-\Omega_{\mathrm{s}} \leqslant 0
\end{aligned}
$$

where $V^{(b)}=\left\{\mathbf{v}=v_{i} \mathbf{e}_{i}: v_{i} \in H^{1}(\Omega)\right.$ with $\mathbf{v}=0$ on $\Gamma_{\mathrm{d}}$ and $\left.\Gamma_{t^{1}}\right\}$.

Equations (20b), (20c) and (21c) are the principle of virtual work. In each case, the displacement field satisfies the equilibrium equation. Equations (20f) and (21f) are the volume constraint of the design domain.

Maximizing $L^{2}\left(\mathbf{u}^{1}\right)$ and minimizing $L^{3}\left(\mathbf{u}^{3}\right)$ simultaneously requires that multi-objective optimization be used. One way to deal with the multi-objective optimization problem is the weighting method formulated as follows:

$$
\begin{aligned}
\underset{\alpha, \beta}{\operatorname{maximize} \theta} & f_{1}=w_{1} L^{2}\left(\mathbf{u}^{1}\right)+w_{2}\left(-L^{3}\left(\mathbf{u}^{3}\right)\right) \\
\text { subject to } \mathbf{t}^{3} & =-\mathbf{t}^{2} \\
a\left(\mathbf{u}^{1}, \mathbf{v}^{1}\right) & =L^{1}\left(\mathbf{v}^{1}\right) \quad \text { for } \mathbf{u}^{1} \in V^{(a)} \forall \mathbf{v}^{1} \in V^{(a)}
\end{aligned}
$$




$$
\begin{aligned}
a\left(\mathbf{u}^{2}, \mathbf{v}^{2}\right) & =L^{2}\left(\mathbf{v}^{2}\right) \quad \text { for } \mathbf{u}^{2} \in V^{(a)} \forall \mathbf{v}^{2} \in V^{(a)} \\
a\left(\mathbf{u}^{3}, \mathbf{v}^{3}\right) & =L^{3}\left(\mathbf{v}^{3}\right) \quad \text { for } \mathbf{u}^{3} \in V^{(a)} \forall \mathbf{v}^{3} \in V^{(b)} \\
0 & \leqslant \alpha \leqslant \alpha_{\text {upp }}<1 \\
0 & \leqslant \beta \leqslant \beta_{\text {upp }}<1 \\
g(\alpha, \beta) & =\int_{\Omega_{\mathrm{d}}}(1-\alpha \beta) \mathrm{d} \Omega-\Omega_{\mathrm{s}} \leqslant 0
\end{aligned}
$$

where $w_{1}$ and $w_{2}$ are given weighting coefficients such that $w_{1} \geqslant 0, w_{2} \geqslant 0, w_{1}+w_{2}=1$.

Since it is possible that $L^{2}\left(\mathbf{u}^{1}\right)$ can go to infinity, and $L^{3}\left(\mathbf{u}^{3}\right)$ must have a finite value, $f_{1}$ may also go to infinity if $w_{1}$ and $w_{2}$ are fixed during the optimization process. To overcome this ill-conditioned problem, weighting coefficients must be adequately adjusted during this process.

A proper alternative way to formulate the multi-objective function is using a ratio rather than linear combination of the two objective functions:

$$
\begin{aligned}
\underset{\alpha, \beta \text { and } \theta}{\operatorname{maximize}} f_{2} & =\frac{L^{2}\left(\mathbf{u}^{1}\right)}{L^{3}\left(\mathbf{u}^{3}\right)} \\
\text { subject to } \mathbf{t}^{3} & =-\mathbf{t}^{2} \\
a\left(\mathbf{u}^{1}, \mathbf{v}^{1}\right) & =L^{1}\left(\mathbf{v}^{1}\right) \quad \text { for } \mathbf{u}^{1} \in V^{(a)} \forall \mathbf{v}^{1} \in V^{(a)} \\
a\left(\mathbf{u}^{2}, \mathbf{v}^{2}\right) & =L^{2}\left(\mathbf{v}^{2}\right) \quad \text { for } \mathbf{u}^{2} \in V^{(a)} \forall \mathbf{v}^{2} \in V^{(a)} \\
a\left(\mathbf{u}^{3}, \mathbf{v}^{3}\right) & =L^{3}\left(\mathbf{v}^{3}\right) \quad \text { for } \mathbf{u}^{3} \in V^{(\mathbf{b})} \forall \mathbf{v}^{3} \in V^{(\mathbf{b})} \\
0 & \leqslant \alpha \leqslant \alpha_{\text {upp }}<1 \\
0 & \leqslant \beta \leqslant \beta_{\text {upp }}<1 \\
g(\alpha, \beta) & =\int_{\Omega_{\mathrm{d}}}(1-\alpha \beta) \mathrm{d} \Omega-\Omega_{\mathrm{s}} \leqslant 0
\end{aligned}
$$

Equation (23a) can be interpreted as the measurement of the efficiency of the compliant mechanisms based on the strain energy. If this value is large, it means that this compliant mechanism can work well and have sufficient rigidity. However, even if one compliant mechanism has two times as much mutual mean compliance and mean compliance as another compliant mechanism, we interpret both of them as having the same efficiency based on the measurement of the strain energy.

The variations of the multi-objective functions $f_{1}$ and $f_{2}$ are described in the following equations:

$$
\begin{aligned}
& \delta f_{1}=w_{1} \delta L^{2}\left(\mathbf{u}^{1}\right)+w_{2}\left(-\delta L^{3}\left(\mathbf{u}^{3}\right)\right) \\
& \delta f_{2}=\frac{1}{L^{3}\left(\mathbf{u}^{3}\right)} \delta L^{2}\left(\mathbf{u}^{1}\right)+\frac{L^{2}\left(\mathbf{u}^{1}\right)}{L^{3}\left(\mathbf{u}^{3}\right)^{2}}\left(-\delta L^{3}\left(\mathbf{u}^{3}\right)\right)
\end{aligned}
$$


Comparing equation (24) with equation (25), the equivalent weighting coefficients $\tilde{w}_{1}$ of the kinematic function $L^{2}\left(\mathbf{u}^{1}\right)$ and $\tilde{w}_{2}$ of the structural function $L^{3}\left(\mathbf{u}^{3}\right)$, with respect to the small perturbation of the objective functions, are obtained by

$$
\tilde{w}_{1}=\frac{L^{3}\left(\mathbf{u}^{3}\right)}{L^{2}\left(\mathbf{u}^{1}\right)+L^{3}\left(\mathbf{u}^{3}\right)}, \quad \tilde{w}_{2}=\frac{L^{2}\left(\mathbf{u}^{1}\right)}{L^{2}\left(\mathbf{u}^{1}\right)+L^{3}\left(\mathbf{u}^{3}\right)}
$$

Note that equivalent weighting coefficients $\tilde{w}_{1}$ and $\tilde{w}_{2}$ are normalized by 1 , and that they are equivalent to the weighting coefficient of equation (22a) in the sense of the derivative of the objective function, or within the small perturbation of the objective function. Equation (26) implies that the equivalent weighting coefficient $\tilde{w}_{2}$ of the structural function $L^{3}\left(\mathbf{u}^{3}\right)$ becomes larger as $L^{2}\left(\mathbf{u}^{1}\right)$ increases; if $L^{2}\left(\mathbf{u}^{1}\right)$ tends to infinity, its equivalent weighting coefficient $\tilde{w}_{1}$ automatically decreases and prevents the problem from being ill-conditioned.

The Lagrangian, $\mathscr{L}_{1}$, of the multi-optimization problem defined by equations $(22 \mathrm{a})-(22 \mathrm{~h})$ is formulated as

$$
\mathscr{L}_{1}=-\left\{w_{1} L^{2}\left(\mathbf{u}^{1}\right)+w_{2}\left(-L^{3}\left(\mathbf{u}^{3}\right)\right)\right\}+\lambda_{1} g(\alpha, \beta)+\lambda_{10}(-A)+\lambda_{11}\left(A-A_{\text {upp }}\right)
$$

where $\lambda_{1}, \lambda_{10}$, and $\lambda_{11}$ are the Lagrange multipliers, $A$ is either $\alpha$ or $\beta$ and $A_{\text {upp }}$ is the upper bound of either $\alpha$ or $\beta$. Note that the negative sign is assigned to the objective function to derive the standard KKT-conditions in which the objective function is to be minimized. The KKT-conditions in this case are written as follows:

$$
\begin{aligned}
\frac{\partial \mathscr{L}_{1}}{\partial A}=-w_{1} \frac{\partial L^{2}\left(\mathbf{u}^{1}\right)}{\partial A}-w_{2}\left(-\frac{\partial L^{3}\left(\mathbf{u}^{3}\right)}{\partial A}\right)+\lambda_{1} \frac{\partial g(\alpha, \beta)}{\partial A}-\lambda_{10}+\lambda_{11} & =0 \\
\frac{\partial \mathscr{L}_{1}}{\partial \theta}=-w_{1} \frac{\partial L^{2}\left(\mathbf{u}^{1}\right)}{\partial \theta}-w_{2}\left(-\frac{\partial L^{3}\left(\mathbf{u}^{3}\right)}{\partial \theta}\right) & =0 \\
g(\alpha, \beta) & \leqslant 0 \\
0 \leqslant A & \leqslant A_{\text {upp }} \\
w_{1} & \geqslant 0 \\
w_{2} & \geqslant 0 \\
\lambda_{1} & \geqslant 0 \\
\lambda_{10} & \geqslant 0 \\
\lambda_{11} & \geqslant 0 \\
\lambda_{1} g(\alpha, \beta) & =0 \\
\lambda_{10}\left(-A_{1}\right. & =0 \\
\lambda_{11}\left(A-A_{\mathrm{upp}}\right) & =0
\end{aligned}
$$


Similarly, the Lagrangian, $\mathscr{L}_{2}$, of the multi-optimization problem defined by equations (23a)-(23h) is formulated as

$$
\mathscr{L}_{2}=-\frac{L^{2}\left(\mathbf{u}^{1}\right)}{L^{3}\left(\mathbf{u}^{3}\right)}+\lambda_{2} g(\alpha, \beta)+\lambda_{20}(-A)+\lambda_{21}\left(A-A_{\text {upp }}\right)
$$

where $\lambda_{2}, \lambda_{20}$ and $\lambda_{21}$ are the Lagrange multipliers, $A$ is either $\alpha$ or $\beta$, and $A_{\text {upp }}$ is the upper bound of either $\alpha$ or $\beta$. Note that in this case the negative sign is also assigned to the objective function to derive the standard KKT-conditions. The KKT-conditions are written as follows:

$$
\begin{aligned}
\frac{\partial \mathscr{L}_{2}}{\partial A}=-\frac{1}{L^{3}\left(\mathbf{u}^{3}\right)} \frac{\partial L^{2}\left(\mathbf{u}^{1}\right)}{\partial A}-\frac{L^{2}\left(\mathbf{u}^{1}\right)}{L^{3}\left(\mathbf{u}^{3}\right)^{2}}\left(-\frac{\partial L^{3}\left(\mathbf{u}^{3}\right)}{\partial A}\right)+\lambda_{2} \frac{\partial g(\alpha, \beta)}{\partial A}-\lambda_{20}+\lambda_{21} & =0 \\
\frac{\partial \mathscr{L}_{2}}{\partial A}=-\frac{1}{L^{3}\left(\mathbf{u}^{3}\right)} \frac{\partial L^{2}\left(\mathbf{u}^{1}\right)}{\partial \theta}-\frac{L^{2}\left(\mathbf{u}^{1}\right)}{L^{3}\left(\mathbf{u}^{3}\right)^{2}}\left(-\frac{\partial L^{3}\left(\mathbf{u}^{3}\right)}{\partial \theta}\right) & =0 \\
g(\alpha, \beta) & \leqslant 0 \\
0 \leqslant A & \leqslant A_{\text {upp }} \\
\lambda_{2} & \geqslant 0 \\
\lambda_{20} & \geqslant 0 \\
\lambda_{21} & \geqslant 0 \\
\lambda_{2} g(\alpha, \beta) & =0 \\
\lambda_{20}(-A) & =0 \\
\lambda_{21}\left(A-A_{\text {upp }}\right) & =0
\end{aligned}
$$

In general, one optimal solution is obtained in the case of the single objective problem. However, in the case of the multi-objective problem, there exists a series of optimal solutions which is called a set of Pareto optima, or noninferior solutions. On the curve of the Pareto optimal set, any further improvement in any objective function requires a worsening of at least one other objective function. As Koski ${ }^{21}$ explained, if $\mathbf{x}$ is the design vector belonging to the feasible set, the Pareto optima is defined as follows:

' $\mathbf{x}$ * is Pareto optimal if there exists no feasible vector $\mathbf{x}$ which would decrease some criterion without causing a simultaneous increase in at least one criterion. In scalar optimization, one optimal solution is usually characteristic of the problem, whereas there generally exists a set of Pareto optima as a solution to a multi-objective problem'.

The necessary conditions for Pareto optimality of the multi-objective function defined by equation (22a) and the multi-objective function defined by equation (23a) are, respectively, equations (28a)-(281) and equations (30a)-(30j). Furthermore, equations (28a)-(281) and equations (30a)-(30j) are identical for some weighting coefficients in equation (22a) if inequality (31) is satisfied. Therefore, if an optimal solution is obtained by equation (23a), this is also one of the optimal solutions of the objective function defined by equation (22a) with some weighting coefficients. This implies that the optimal solution of the objective function defined by equation 
(23a) is one of the noninferior solutions of the objective function defined by equation (22a).

$$
\frac{1}{L^{3}\left(\mathbf{u}^{3}\right)} \geqslant 0, \quad \frac{L^{2}\left(\mathbf{u}^{1}\right)}{L^{3}\left(\mathbf{u}^{3}\right)^{2}} \geqslant 0
$$

Thus, the optimality condition for the multi-objective problem defined by equations (23a)-(23h) is confirmed.

Moreover, the optimization problem for only the structural function is considered. If the only design variables $\alpha$ and $\beta$ are taken into account, the Lagrangian, $\mathscr{L}_{3}$, is defined by

$$
\mathscr{L}_{3}=L^{3}\left(\mathbf{u}^{3}\right)+\lambda_{3} g(\alpha, \beta) \text { for } 0<A<A_{\text {upp }}
$$

where $\lambda_{3}$ is the Lagrange multiplier, $A$ is either $\alpha$ or $\beta$, and $A_{\text {upp }}$ is the upper bound of either $\alpha$ or $\beta$. $\mathrm{Ma}^{17}$ studied the characteristics of the minimum mean compliance problem for the structural function, and noted that the volume constraint of equation (21f) is active at the optimum, and $\lambda_{3}$ is positive at the optimum in equation (33),

$$
\lambda_{3}=\frac{\partial L^{3}\left(\mathbf{u}^{3}\right) / \partial A}{\partial g(\alpha, \beta) / \partial A}>0 \quad \text { for } 0<A<A_{\text {upp }}
$$

where $A$ is either $\alpha$ or $\beta$, and $A_{\text {upp }}$ is the upper bound of either $\alpha$ or $\beta$.

If $L^{2}\left(\mathbf{u}^{1}\right) \gg L^{3}\left(\mathbf{u}^{3}\right)>0$ at the optimum, using equations (30a) and (33), we have

$$
\lambda_{2}=-\frac{\frac{1}{L^{3}\left(\mathbf{u}^{3}\right)^{2}}\left\{-\mathbf{L}^{3}\left(\mathbf{u}^{3}\right) \partial L^{2}\left(\mathbf{u}^{1}\right) / \partial A+L^{2}\left(\mathbf{u}^{1}\right) \partial L^{3}\left(\mathbf{u}^{3}\right) / \partial A\right\}}{\partial g(\alpha, \beta) / \partial A}>0 \quad \text { for } 0<A<A_{\text {upp }}
$$

where $A$ is either $\alpha$ or $\beta$, and $A_{\text {upp }}$ is the upper bound of either $\alpha$ or $\beta$.

Since the Lagrange multiplier $\lambda_{2}$ is also positive at the optimum, the volume constraint of equation (23h) is also active in the case of the multi-objective optimization problem formulated as in equations (23a)-(23h). This condition guarantees that some compliant mechanism solutions can be obtained. Thus, the multi-objective optimization problem is formulated by equations (23a)-(23h).

Figure 4 shows a flowchart of the optimization procedure. There are four main steps in the per-iteration loop of the optimization. First, the homogenized coefficients are calculated. For interpolation of the microscopic design variables $\alpha$ and $\beta$, Bezier curves are used. Next, FEM is used to calculate the sensitivities of the objective function and constraints with respect to $\alpha$ and $\beta$ in the unit cell of the microstructure (Figure 2). In the FEM, two-dimensional four-node isoparametric and full integration elements with bilinear shape functions are used. These sensitivities are used in the optimization phase.

The optimality criteria method has been successful in many structural optimal design problems. However, $\mathrm{Ma}^{17}$ pointed out that it is difficult to construct the heuristics for a general optimization problem to evaluate optimality if the objective function is not convex. To overcome this problem, Ma employed a convex approximation. However, it is also difficult to construct the updating scheme for the complicated objective function since this updating scheme is more or less based on the heuristics. On the other hand, Sequential Linear Programming (SLP) is easily implemented even in the case of the complicated objective function, and can be said to be the 


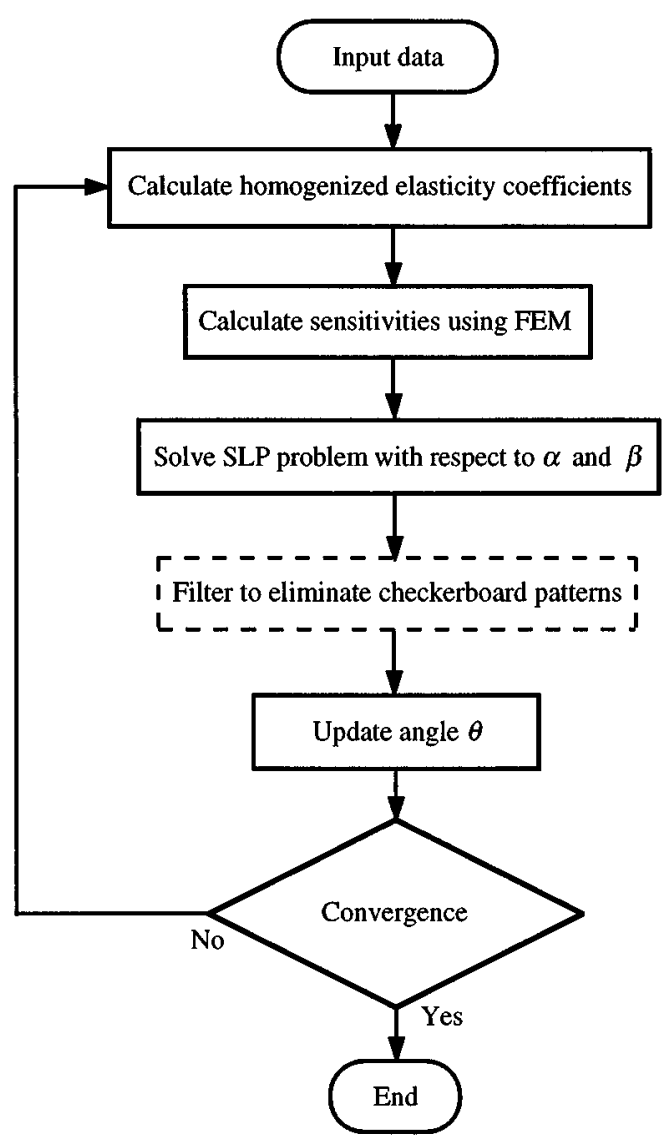

Figure 4. Flowchart of optimization procedure

simplest convex approximation. Based on this reason, SLP is used to solve the optimization problem. An advantage of using SLP is that it can handle over 10000 design variables, although fast convergence cannot be expected. SLP is used to solve for microscale variables $\alpha$ and $\beta$. In the linearized optimization procedure, the simplex method is used. There are several methods ${ }^{22,23}$ to set the move limits for SLP. Since the topology optimization problem has many simple constraints, the move limits are usually set to 5 to $15 \%$ of the original values of design variables. In this study, the move limits are set to $10 \%$ of the original values, and are decreased to lower limits when the objective function reaches the proximity of the optimal value. In the linearized optimization problem, a package of the simplex method, DSPLP from the SLATEC library, ${ }^{24}$ is used.

The angle $\theta$ in the unit cell must be updated to maximize the multi-objective function (23a) as well. However, $\theta$ is practically updated to minimize the objective function (21a) because the principal direction of the stress is almost the same as the direction in which the objective function (23a) is maximized. This principal direction is identical to the direction in which the objective function (21a) is minimized based on Suzuki and Kikuchi ${ }^{14}$ and Pedersen. ${ }^{25}$ If the convergence criteria is satisfied, then the process is terminated. 
The sensitivities of the objective function, $f_{2}$, and the volume constraint, $g(\alpha, \beta)$, required in SLP are obtained by

$$
\frac{\partial f_{2}}{\partial A}=\frac{-\int_{\Omega} \varepsilon\left(\mathbf{u}^{2}\right)^{\mathrm{T}} \frac{\partial \mathbf{E}}{\partial A} \varepsilon\left(\mathbf{u}^{1}\right) \mathrm{d} \Omega a\left(\mathbf{u}^{3}, \mathbf{u}^{3}\right)+\int_{\Omega} \varepsilon\left(\mathbf{u}^{3}\right)^{\mathrm{T}} \frac{\partial \mathbf{E}}{\partial A} \varepsilon\left(\mathbf{u}^{3}\right) \mathrm{d} \Omega a\left(\mathbf{u}^{1}, \mathbf{u}^{2}\right)}{a\left(\mathbf{u}^{3}, \mathbf{u}^{3}\right)^{2}}
$$

where $A$ is either $\alpha$ or $\beta$, and

$$
\begin{aligned}
& \frac{\partial g(\alpha, \beta)}{\partial \alpha}=\int_{\Omega_{\mathrm{d}}}(-\beta) \mathrm{d} \Omega \\
& \frac{\partial g(\alpha, \beta)}{\partial \beta}=\int_{\Omega_{\mathrm{d}}}(-\alpha) \mathrm{d} \Omega
\end{aligned}
$$

Checkerboard patterns often appear in the optimal configuration when using the homogenization method with the displacement-based finite element method. As Díaz and Sigmund ${ }^{26}$ explained, if four-node isoparametric elements with bilinear shape functions are used, the pattern of checkerboard shown in Figure 5(b) is artificially stiffer than the pattern in which each element has the uniform density of $1 / 2$ as shown in Figure 5(c). To overcome this problem, the modified filtering scheme based on Bendsøe's method ${ }^{27}$ is developed. The algorithm of this filtering scheme is described as follows:

(1) Construct one group consisting of the four contiguous finite elements as shown in Figure 5(a). The shape of all four contiguous finite elements is assumed to be the same. The microscopic variables $\alpha$ and $\beta$ of the ith element $(i=1, \ldots, 4)$ are described as $\alpha_{i}$ and $\beta_{i}$, respectively. The density of the $i$ th element is calculated by

$$
\rho_{i}=1-\alpha_{i} \beta_{i} \quad(i=1, \ldots, 4)
$$

(2) Search for the maximum value and the minimum value of $\rho_{i}(i=1, \ldots, 4)$. The maximum value is set to $\rho_{\max }$, and the minimum value is set to $\rho_{\min }$.

\begin{tabular}{|l|l|}
\hline$\rho_{1}$ & $\rho_{3}$ \\
\hline$\rho_{2}$ & $\rho_{4}$ \\
\hline
\end{tabular}

(a)

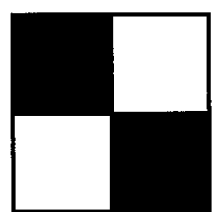

$\rho_{1}=\rho_{4}=1$

$\rho_{2}=\rho_{3}=0$

(b)

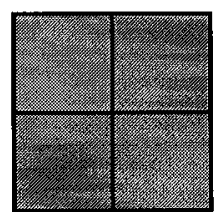

$\rho_{1}=\rho_{2}=1 / 2$

$\rho_{3}=\rho_{4}=1 / 2$

(c)

Figure 5. A checkerboard pattern 
(3) Calculate the filtered density $\bar{\rho}_{i}(i=1, \ldots, 4)$ using the following equations:

$$
\begin{aligned}
& \bar{\rho}_{1}=\frac{1}{4}\left(3 \rho_{1}+\rho_{2}+\rho_{3}-\rho_{4}\right) \\
& \bar{\rho}_{2}=\frac{1}{4}\left(\rho_{1}+3 \rho_{2}-\rho_{3}+\rho_{4}\right) \\
& \bar{\rho}_{3}=\frac{1}{4}\left(\rho_{1}-\rho_{2}+3 \rho_{3}+\rho_{4}\right) \\
& \bar{\rho}_{4}=\frac{1}{4}\left(-\rho_{1}+\rho_{2}+\rho_{3}+3 \rho_{4}\right)
\end{aligned}
$$

(4) Search for the maximum value and the minimum value of $\bar{\rho}_{i}(i=1, \ldots, 4)$. The maximum value is set to $\bar{\rho}_{\max }$, and the minimum value is set to $\bar{\rho}_{\text {min }}$.

(5) If the following inequalities are satisfied in each group consisting of the four contiguous finite elements, replace the density of each element with $\bar{\rho}_{i}$. Otherwise, the density is not filtered.

$$
\bar{\rho}_{\max }<\rho_{\max } \text { and } \rho_{\min }>\rho_{\min }
$$

Note that only if these equalities are satisfied, this group will have a checkerboard pattern.

(6) In the elements in which the density is filtered, calculate the equivalent microscopic variables, $\bar{\alpha}_{i}$ and $\bar{\beta}_{i}$ as follows:

$$
\begin{aligned}
& \bar{\alpha}_{i}=\bar{\gamma}_{i} \alpha_{i} \\
& \bar{\beta}_{i}=\bar{\gamma}_{i} \beta_{i}
\end{aligned}
$$

where

$$
\bar{\gamma}_{i}=\left(\frac{1-\bar{\rho}_{i}}{\alpha_{i} \beta_{i}}\right)^{1 / 2}
$$

If severe checkerboard patterns are recognized in the optimal configuration, this filtering scheme is used to eliminate them. In the flowchart of the optimization procedure shown in Figure 4, the filtering scheme is executed after the SLP procedure.

\section{EXAMPLES}

Three examples are presented here to examine the configuration of the optimal solutions. In all the examples, the properties of the isotropic material correspond to Young's modulus $=100$ and Poisson's ratio $=0 \cdot 3$, and two-dimensional elements are used for the finite element analysis. In all cases, the applied force is assumed to be a unit load.

\section{Example 1. Effect of mesh size}

The first example is used to verify the uniqueness of optimal topology configuration when finite element meshes are uniformly refined, while other conditions are fixed in this example. Figure 6 shows the design domain specified as a 240 by 120 rectangle with a fixed support boundary on the left-hand side. The deflection at point $B$ in the direction of the dummy load is to be maximized when the external force is applied at point $\mathrm{A}$, while the mean compliance at point $\mathrm{B}$ is to be minimized. 


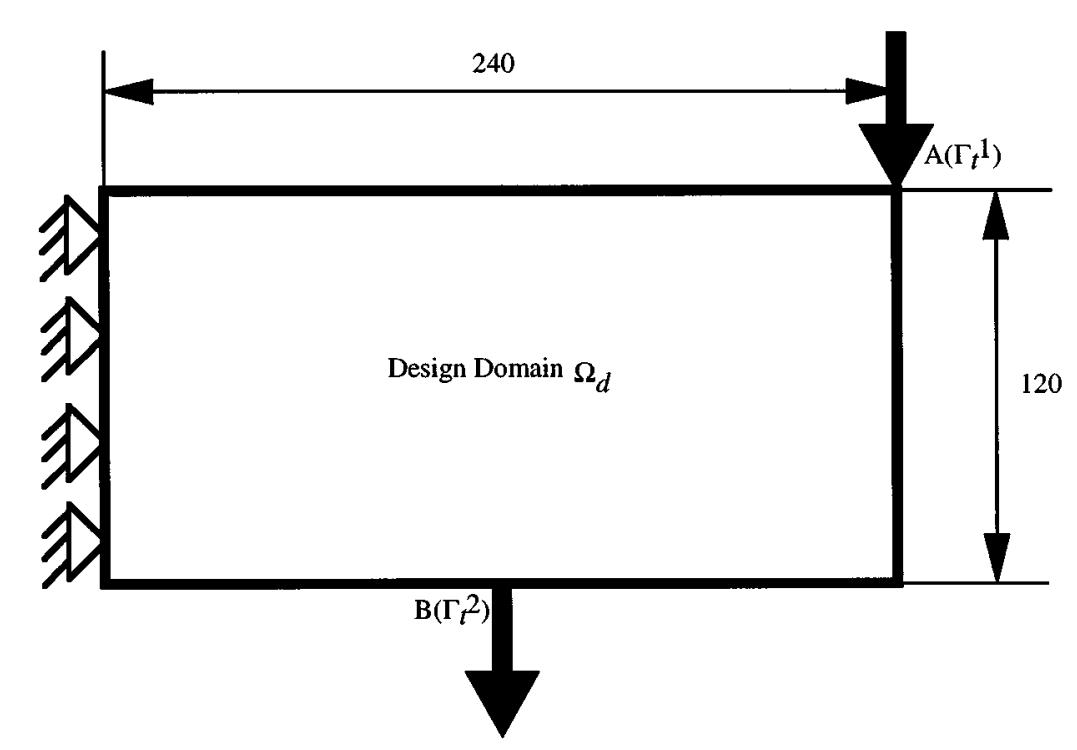

Figure 6. Design domain for Example 1

Three finite element models are utilized for the verification. One is a coarse mesh using $40 \times 20$ finite elements, another is an intermediate mesh using $60 \times 30$ finite elements, and the third is a fine mesh using $80 \times 40$ finite elements. The total volume constraint of the material $\Omega_{\mathrm{s}}$ is considered to be 5760, which is 20 per cent of the volume of the whole design domain. The initial value of microscopic design variables $\alpha$ and $\beta$ is 0.95 , and that of $\theta$ is 0.0 in all elements.

Figure 7 illustrates the convergence history of the optimization process in the case of $60 \times 30$ finite elements. Notice that the mutual mean compliance defined by equation (20a) increases and the mean compliance defined by equation (21a) decreases simultaneously (Figure 7(a)), while the objective function is maximized (Figure 7(b)). Also note that the volume constraint is active at the optimal point (Figure $7(\mathrm{~b})$ ), and that the equivalent weighting coefficient, $\tilde{w}_{1}$, of the mutual mean compliance is close to zero when the objective function is maximized (Figure 7(c)).

Figure 8 shows the results of the optimal configurations with respect to the various meshes. It is clear that the optimal topology configurations using three different mesh sizes are similar, though slight differences in shape are observed in some portions. This means that the optimal topology configuration is not affected by the size of meshes.

\section{Example 2. Compliant gripper and effect of volume constraint}

This example illustrates the design of a compliant gripper, and is used to examine the relation between the optimal topology configurations and the total volume constraint of material $\Omega_{\mathrm{s}}$.

Figure 9 shows a half-view of the design domain where boundary conditions and specifications are as indicated. The function of the gripper is to (1) deform along the direction of the dummy force in order to grasp a workpiece at point B when the external force is applied at point A, and (2) hold the workpiece while the external force is continuously applied. The mutual mean compliance between points $\mathrm{A}$ and $\mathrm{B}$ is maximized while the mean compliance at point $\mathrm{B}$ is minimized in order 

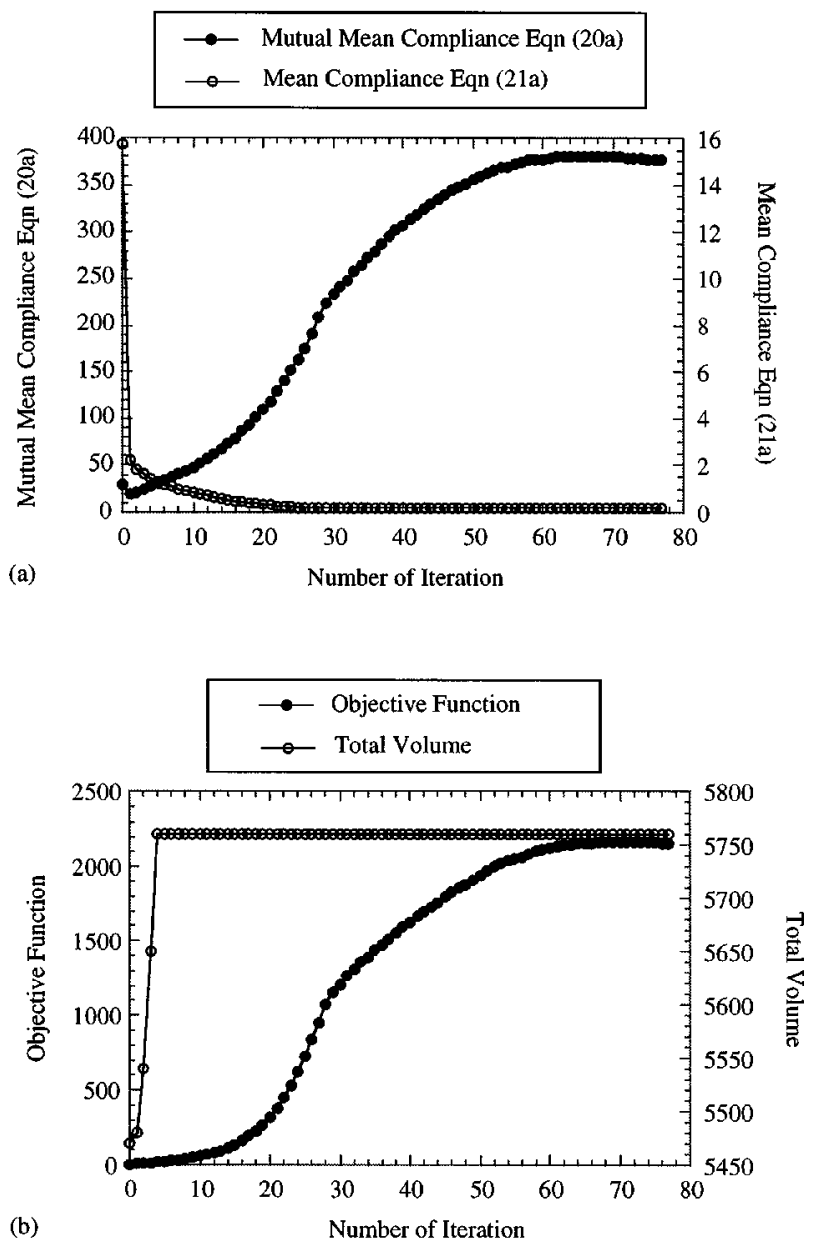

(b)

Number of Iteration

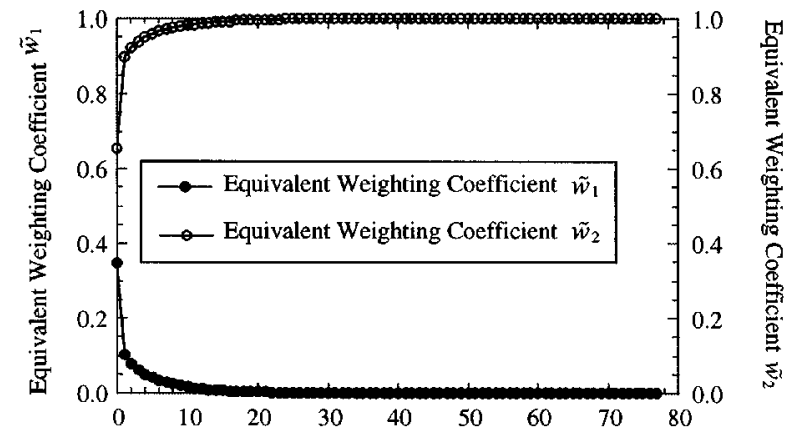

(c)

Number of Iteration

Figure 7. Convergence history $(60 \times 30)$ : (a) mean compliance; (b) objective function and total volume; (c) equivalent weighting coefficients normalized by 1 


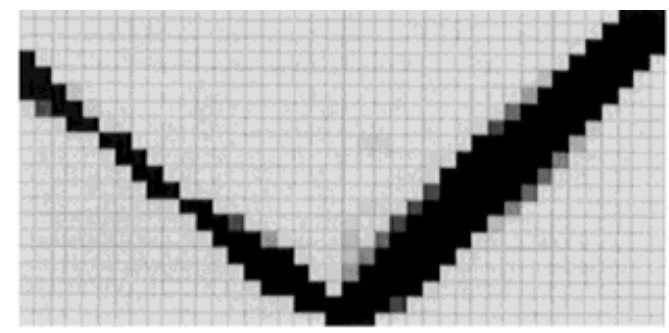

(a)

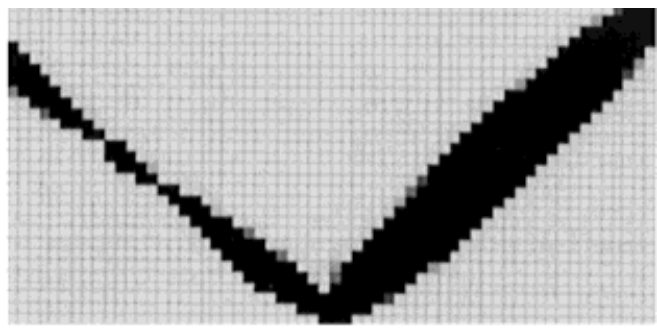

(b)

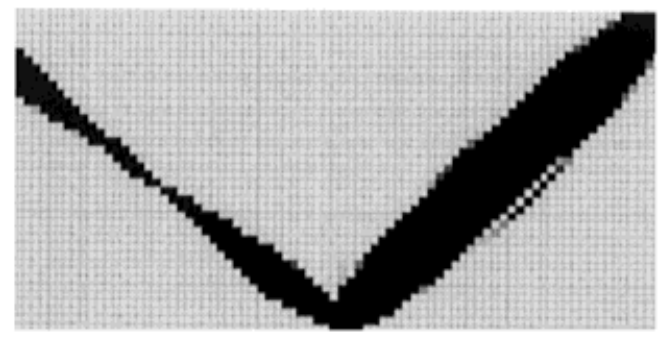

(c)

Figure 8. Optimal configurations using different meshes: (a) optimal configuration $(40 \times 20)$; (b) optimal configuration $(60 \times 30)$; (c) optimal configuration $(80 \times 40)$

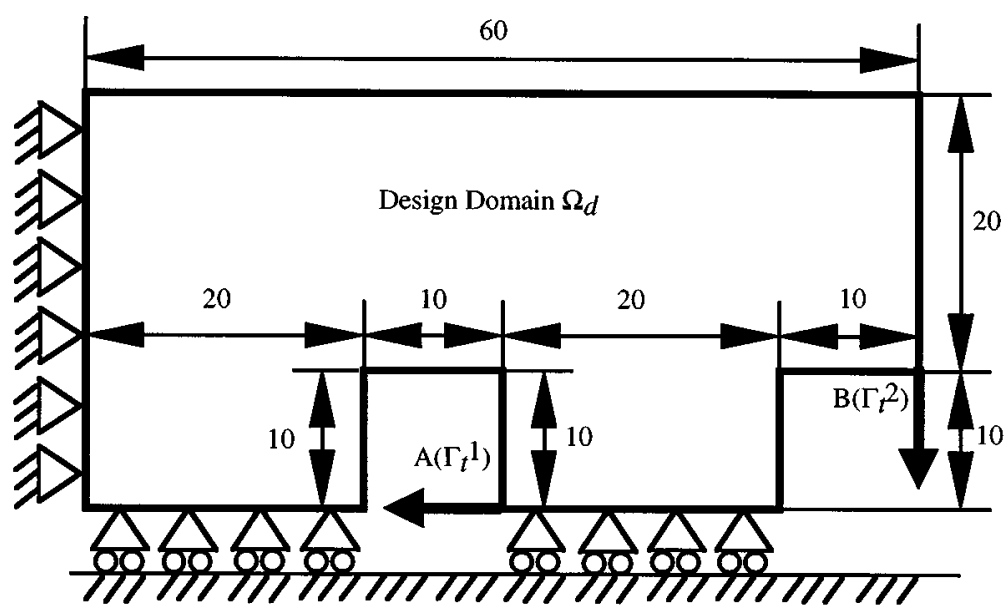

Figure 9. Design domain for compliant gripper

to satisfy the required function. The left side boundary of the design domain is fixed for support of the gripper. The symmetric boundary condition is posed at the bottom side boundary. In this problem, 1600 finite elements are used. The initial value of microscopic design variables $\alpha$ and $\beta$ is $0 \cdot 9$, and that of $\theta$ is $0 \cdot 0$ in all elements. Three values of the total volume constraint $\Omega_{\mathrm{s}}, 160,240$ and 400 , are examined. 
Figure 10 shows the convergence history of the optimization process in the case of $\Omega_{\mathrm{s}}=240$. The mutual mean compliance defined by equation (20a) increases and the mean compliance defined by equation (21a) decreases simultaneously (Figure 10(a)), while the objective function is maximized (Figure 10(b)) in the case of the complex structural design. The volume constraint is active when the objective function has been maximized (Figure 10(b)).

Figure 11 shows the optimal topology configurations for the case of $\Omega_{\mathrm{s}}=160,240$ and 400 . Comparing the case of $\Omega_{\mathrm{s}}=240$ with the case of $\Omega_{\mathrm{s}}=160$, the topology configurations near point A are different. However, the topology configurations in the cases of $\Omega_{\mathrm{s}}=240$ and 400 are almost the same, although a slight difference in the optimal shape is observed.

The material density near point $B$ increases as the total volume constraint increases because the mean compliance defined by equation (21a) decreases. This implies that it is necessary to have sufficient material in order to obtain the rigidity required to hold a workpiece. On the other hand,

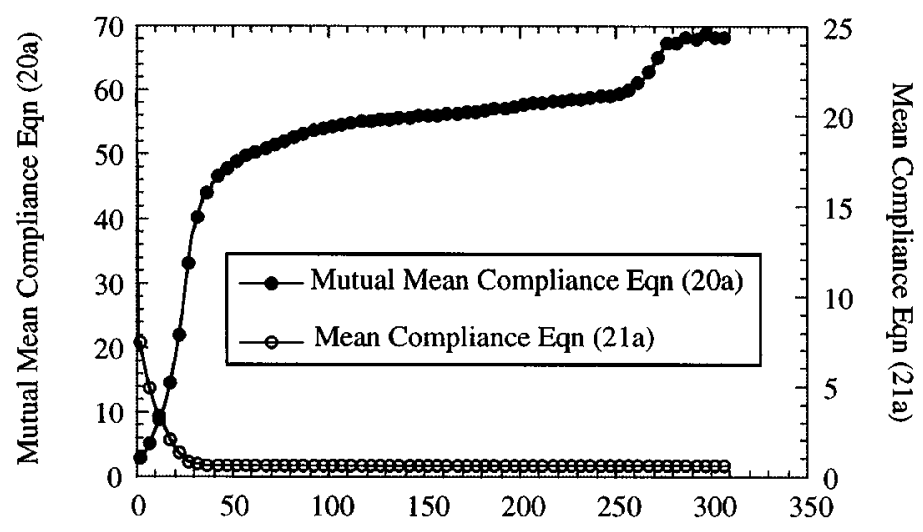

(a)

Number of Iteration

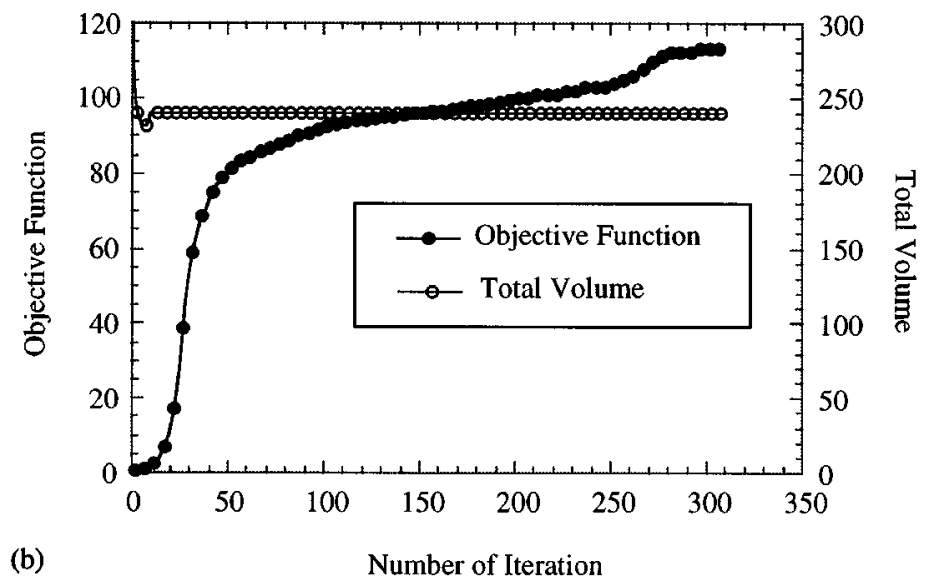

Figure 10. Convergence history $\left(\Omega_{\mathrm{s}}=240\right.$ ): (a) mean compliance; (b) objective function and total volume 


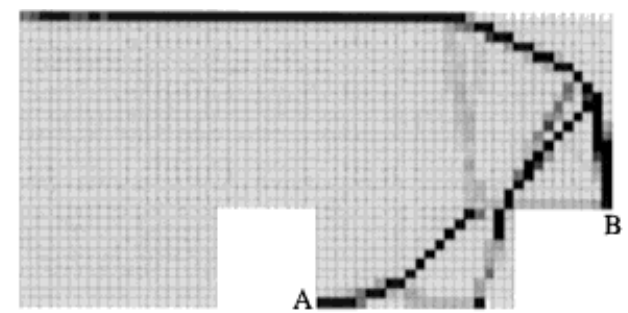

(a)

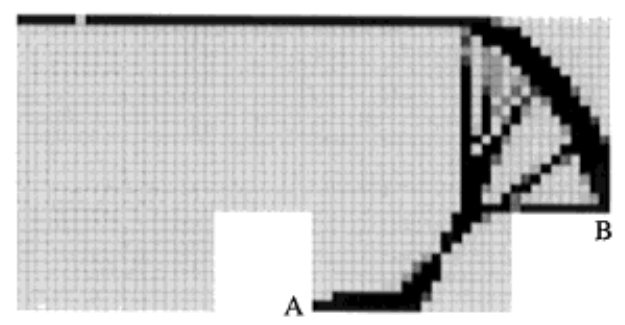

(b)

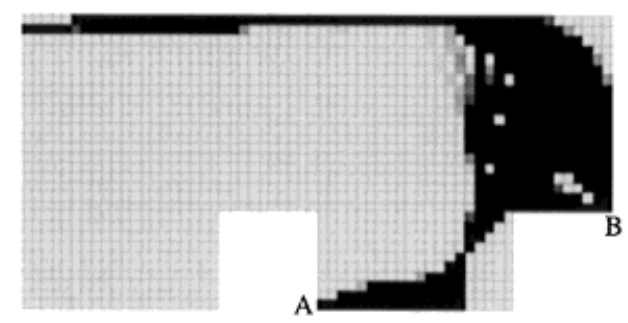

(c)

Figure 11. Optimal configurations with different total volume constraints: (a) optimal configuration $\left(\Omega_{\mathrm{s}}=160\right)$; (b) optimal configuration $\left(\Omega_{\mathrm{s}}=240\right)$; (c) optimal configuration $\left(\Omega_{\mathrm{s}}=400\right)$

the topology configuration on the left side which connects to the fixed boundary portions does not change with respect to the total volume constraint. This implies that this configuration is required for the flexibility of the gripper. In summary, it is likely that the optimal topology configuration is affected by changing the total volume constraint.

\section{Example 3. Compliant clamp and performance of optimal configuration}

This example illustrates the design of a compliant clamp, and is used to examine whether the optimal topology configuration matches the performance of compliant mechanism design. Ordinary nonlinear FEM is used for this analysis. Figure 12 shows a half view of the design domain where boundary conditions are as indicated. The function of the clamp is to (1) hold a workpiece at point $\mathrm{B}$ while the external force at point $\mathrm{A}$ is not applied, and (2) deform to release the workpiece when the external force at point $\mathrm{A}$ is applied. The mean compliance at point $\mathrm{B}$ is minimized while the mutual mean compliance between points $\mathrm{A}$ and $\mathrm{B}$ is maximized in order to satisfy the required function. The bottom side boundary of the design domain is fixed since the clamp is supported at this boundary. The design domain is discretized using 1760 finite elements. The initial value of microscopic design variables $\alpha$ and $\beta$ is 0.90 , and that of $\theta$ is 0.0 in all elements. The total volume constraint of the material $\Omega_{\mathrm{s}}$ is considered to be 352 which is 20 per cent of the volume of the whole design domain.

Figure 13 shows the optimal topology configuration of the compliant clamp. Since checkerboard patterns are observed in this optimal configuration, the filtering scheme is employed to 


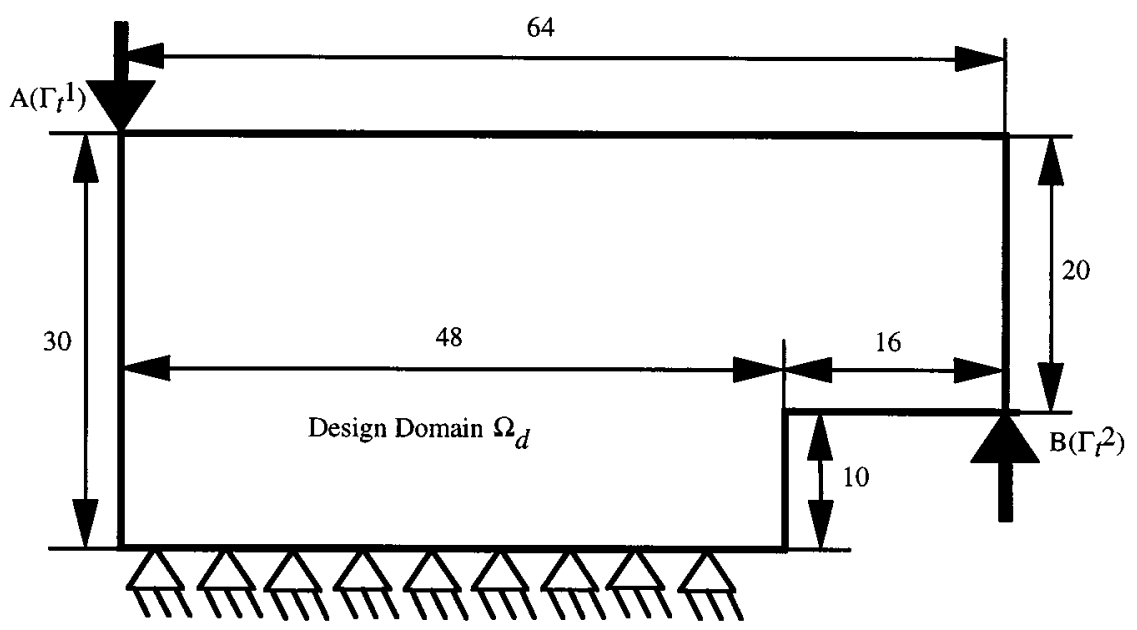

Figure 12. Design domain for compliant clamp

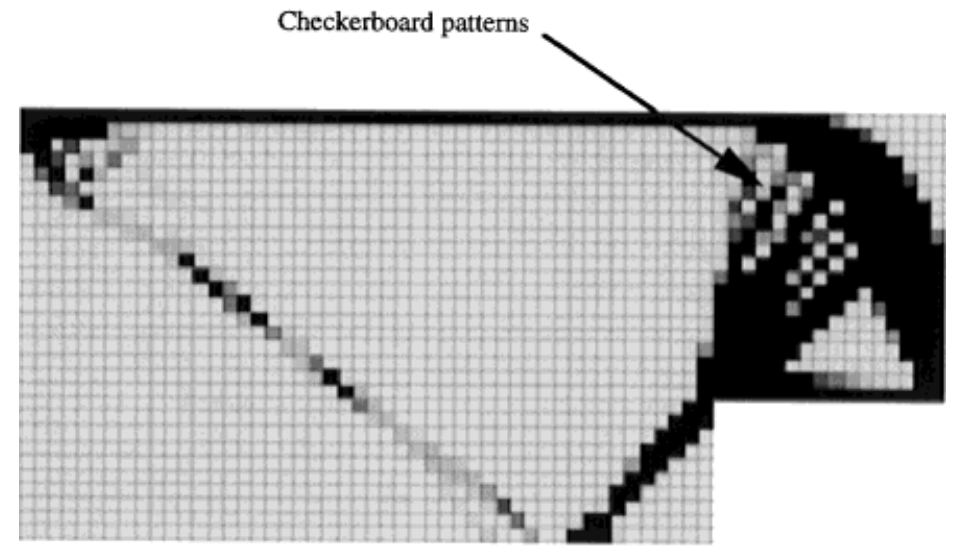

Figure 13. Optimal configuration of compliant clamp

eliminate them. Figure 14 illustrates the convergence history of the optimization process of the clamp design when the filtering scheme is used. The mutual mean compliance defined by equation (20a) increases, and the mean compliance defined by equation (21a) decreases simultaneously (Figure 14(a)), even though the filtering scheme is used. The volume constraint is active when the objective function has been maximized (Figure 14(b)). Figure 15 shows the optimal topology configuration of the compliant clamp using the filtering scheme.

The ordinary finite element model is constructed based on this optimal topology configuration in order to verify the function of the compliant clamp. This model is discretized to 2001 elements with 2318 nodes as shown in Figure 16. The properties of the isotropic material correspond to Young's modulus $=100$ and Poisson's ratio $=0 \cdot 3$. Non-linear commercial code ABAQUS with large displacement option is used for the non-linear analysis. 


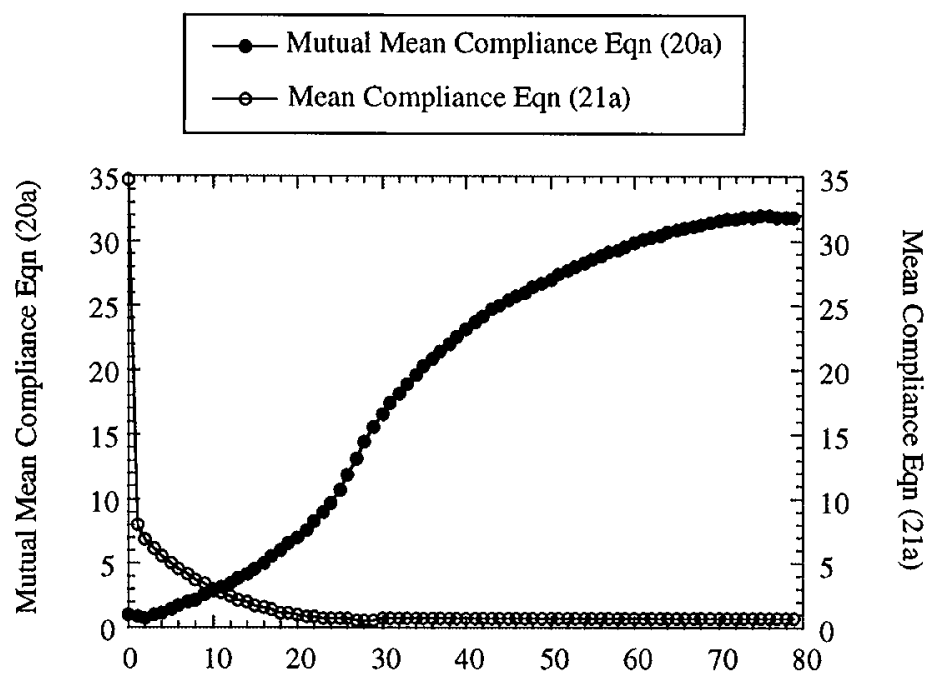

(a)

Number of Iteration

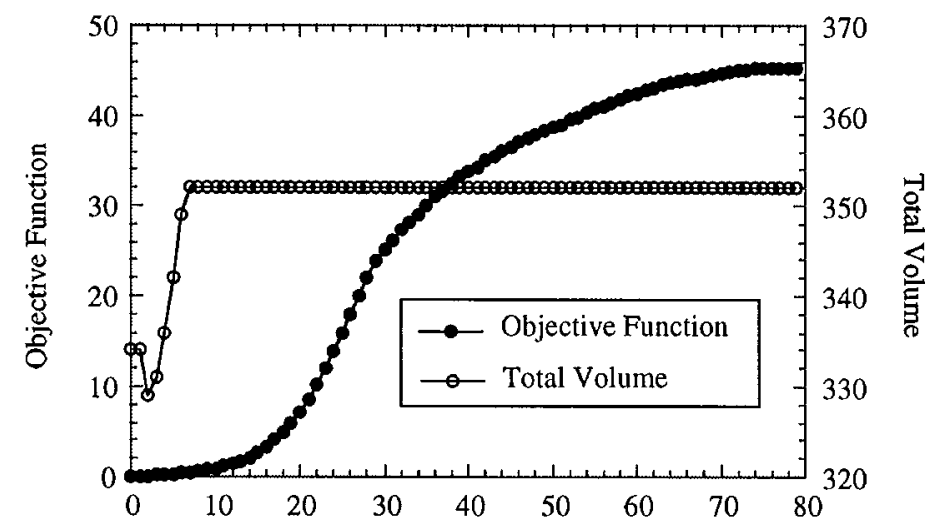

(b)

Number of Iteration

Figure 14. Convergence history of compliant clamp design (Filtering scheme is used): (a) mean compliance; (b) objective function and total volume

Figure 17 shows the deformation, and Figure 18 shows the von-Mises stress distribution of the compliant clamp where an external force of $0 \cdot 1$ unit is applied at point A. It is clear that the compliant clamp deforms according to the problem specifications. That is, the portion around $\mathrm{B}$ is rising in order to release a workpiece when the external force is applied. Note that the stress concentrations are near the midspan of the left-diagonal member and the support positions of the right-diagonal member. The higher stress concentration occurs near support positions of the 


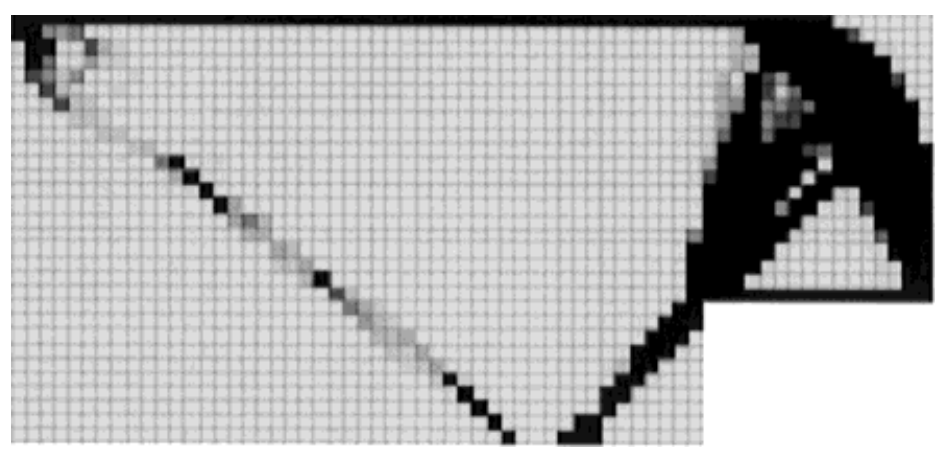

Figure 15. Optimal configuration of compliant clamp (Filtering scheme is used)

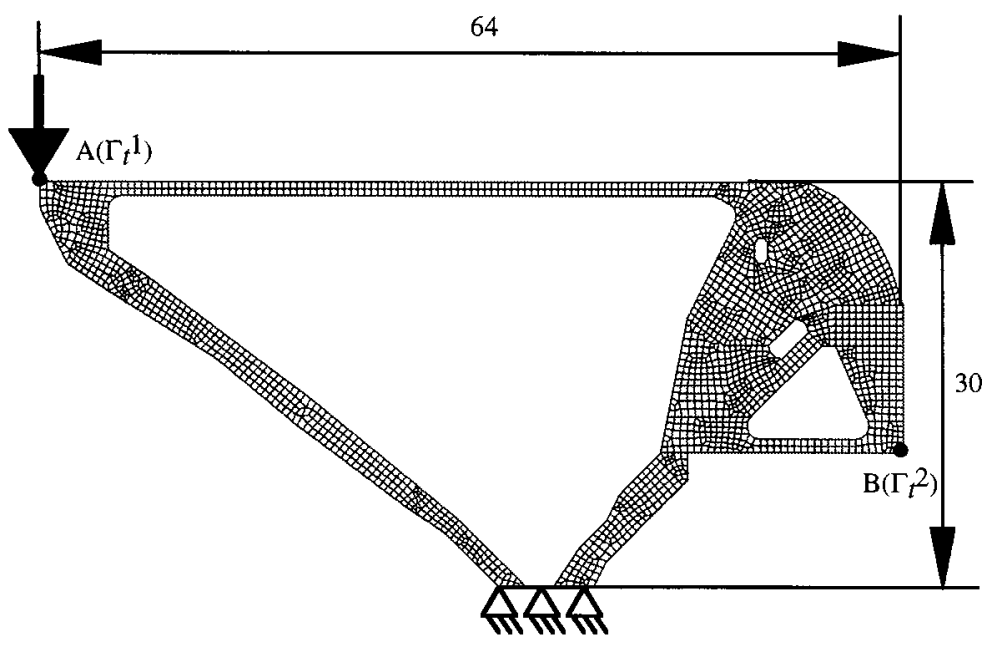

Figure 16. Finite element model

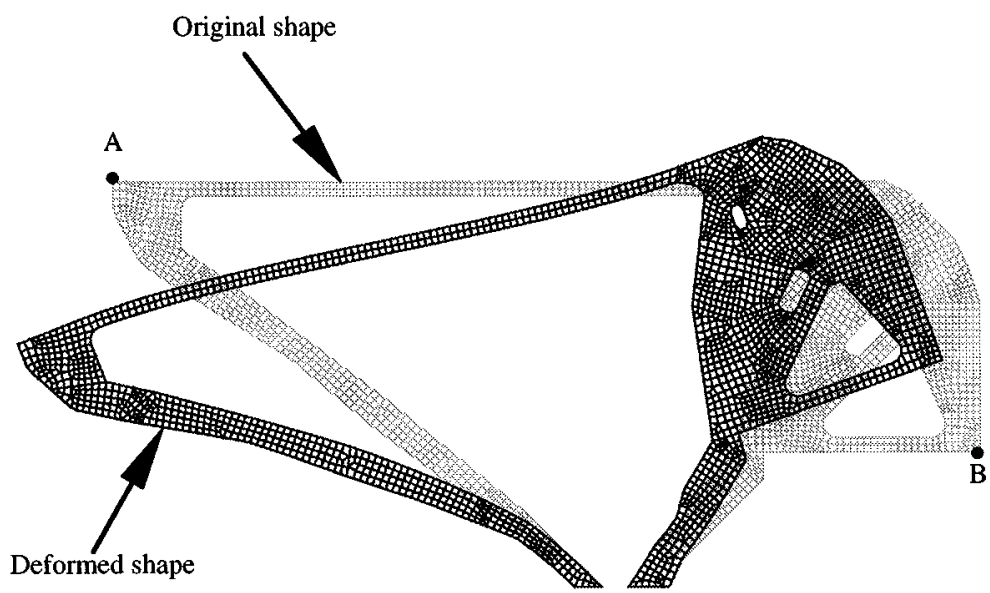

Figure 17. Deformed shape for non-linear analysis 


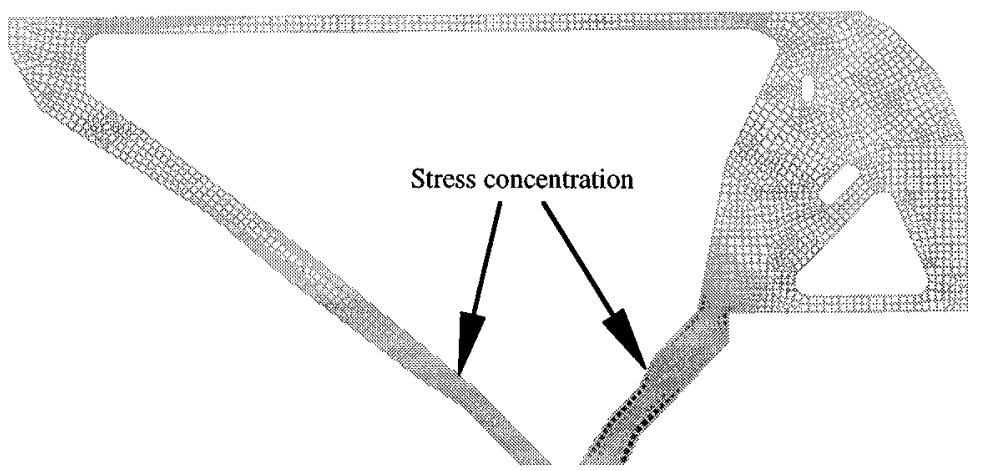

Figure 18. Distribution of von-Mises stress (The darker scale describes the higher stress concentration)

right-diagonal member. This implies that there is a lower probability of fracture except in these positions even though the compliant clamp deforms non-linearly. Therefore, the design of compliant mechanisms can be accomplished with the method proposed in this research.

\section{CONCLUSION}

A topology optimization method considering flexibility has been developed using the homogenization method and applied to the design of compliant mechanisms. Mutual mean compliance was introduced in order to formulate the flexibility of structures, and its sensitivity was obtained for the structural optimization. A new formulation of multi-objective optimization was introduced for the design of compliant mechanisms. An optimization algorithm was constructed using the homogenization method and Sequential Linear Programming (SLP). It has been shown that the optimal topology configuration obtained by the multi-objective formulation is unique, regardless of the number of finite elements. It has also been shown that the optimal topology configuration is affected by a change in the total volume constraint of materials. The additional finite element analysis proved that the final compliant mechanism design satisfies the problem specifications.

\section{ACKNOWLEDGEMENTS}

The authors are supported partially by grant DMI 9622261 from the National Science Foundation and TOYOTA Central R\&D Labs., Inc. They are grateful for this support. At the initial stage of the study on compliant mechanisms, the authors had opportunity to read the dissertation, 'A new design paradigm for micro-electro-mechanical systems \& investigation on the compliant mechanisms synthesis' by Ananthasuresh, Gondi Kondaiah, Ph.D. (1994) in the University of Michigan. They appreciate this opportunity to study of the pioneering work on this subject.

\section{REFERENCES}

1. A. Midha, T. W. Norton and L. L. Howell, 'On the nomenclature and classification of compliant mechanisms: the components of mechanisms', Proc. ASME Design Engineering Technical Conf., Arizona, Vol. 47, 1992, pp. $13-16$.

2. M. I. Frecker, N. Kikuchi and S. Kota, 'Optimal synthesis of compliant mechanisms to satisfy kinematic and structural requirements-preliminary results', Proc. ASME Design Engineering Technical Conf., Irvine, Vol. 96DETC/DAC-1497, 1996, pp. 18-22. 
3. I. Her and A. Midha, 'A compliance number concept for compliant mechanisms, and type synthesis', ASME J. Mechanisms Transmissions Automat. Design, 109, 348-355 (1986).

4. M. D. Murphy, A. Midha and L. L. Howell, 'The topological synthesis of compliant mechanisms', Proc. 3 National Conf. of Appl. Mech. and Robot., Ohio, Vol. 2, Paper No. 99, 1993.

5. L. L. Howell and A. Midha, 'A method for the design of compliant mechanisms with small-length flexural pivots', ASME J. Mech. Des., 116, 280-289 (1994).

6. L. L. Howell and A. Midha, 'A loop-closure theory for the analysis and synthesis of compliant mechanisms', ASME J. Mech. Des., 118, 121-125 (1996).

7. L. L. Howell and A. Midha, 'Evaluation of equivalent spring stiffness for use in pseudo-rigid-body model of large-deflection compliant mechanisms', ASME J. Mech. Des., 118, 126-131 (1996).

8. O. Sigmund, 'Some inverse problem in topology design of materials and mechanisms', Proc. IUTAM Symp. on Optimization of Mechanical Systems, Stuttgart, Germany, 1995, pp. 26-31.

9. U. D. Larsen, O. Sigmund and S. Bouswstra, 'Design and fabrication of compliant micromechanisms and structures with negative Possion's ratio', Proc. IEEE 9th Annual Int. Workshop on Micro Electro Mechanical Systems, San Diego, 1996, pp. 11-15.

10. M. P. Bendsøe and N. Kikuchi, 'Generating optimal topologies in structural design using a homogenization method', Comput. Meth. Appl. Mech. Engng., 71, 197-224 (1988).

11. K. T. Cheng and N. Olhoff, 'An investigation concerning optimal design of solid elastic plates', Int. J. Solids Struct., 17, 305-323 (1981).

12. R. V. Kohn and G. Strang, 'Optimal design and relaxation of variational problems', Comm. Pure Appl. Math., 39, 1-25 (Part 1), 139-182 (Part 2) and 353-377 (Part 3) (1986).

13. G. I. N. Rozvany, T. G. Ong, W. T. Seto, N. Olhoff and M. P. Bendsøe, 'Least-weight design of perforated plates', Int. J. Solids Struct., 23, 521-536 (Part 1), 537-550 (Part 2) (1987).

14. K. Suzuki and N. Kikuchi, 'A homogenization method for shape and topology optimization', Comput. Meth. Appl. Mech. Engng., 93, 291-318 (1991).

15. A. Díaz and N. Kikuchi, 'Solutions to shape and topology eigenvalue optimization problems using a homogenization method', Int. J. Numer. Meth. Engng., 35, 1487-1502 (1992).

16. Z.-D. Ma, N. Kikuchi, H.-C. Cheng and I. Hagiwara, 'Topological optimization technique for free vibration problems', ASME J. Appl. Mech., 62, 200-207 (1995).

17. Z.-D. Ma, N. Kikuchi and H.-C. Cheng, 'Topological design for vibrating structures', Comput. Meth. Appl. Mech. Engng., 121, 250-280 (1995).

18. G. K. Ananthasuresh, S. Kota and Y. Gianchandani, 'Systematic synthesis of microcompliant mechanisms-preliminary results', Proc. 3rd National Conf. of Appl. Mech. and Robot., Ohio, Vol. 2, Paper No. 82, 1993.

19. R. T. Shield and W. Prager, 'Optimal structural design for given deflection', J. Appl. Math. Phys. ZAMP, 21, 513-523 (1970).

20. N. Huang, 'On principle of stationary mutual complementary energy and its application to optimal structural design', J. Appl. Math. Phys. ZAMP, 22, 609-620 (1971).

21. J. Koski, 'Defectiveness of weighting method in multicriterion optimization of structures', Comm. Appl. Numer. Meth., 1, 333-337 (1985).

22. T.-Y. Chen, 'Calculation of the move limits for the sequential linear programming method', Int. J. Numer. Meth. Engng., 36, 2661-2679 (1993).

23. H. L. Thomas, G. N. Vanderplaats and Y.-K. Shyy, 'A study of move limit adjustment strategies in the approximation concept approach to structural synthesis', Proc. 4th AIAA/USAF/NASA/OAI Symp. on Multidisciplinary Analysis and Optimization, Cleveland, 1992, pp. 507-512.

24. R. Hanson and K. Hiebert, 'A sparse linear programming subprogram', Technical Report SAND81-0297, Sandia National Laboratories, 1981.

25. P. Pedersen, 'On optimal orientation of orthotopic materials', Struct. Optim., 1, 101-106 (1989).

26. A. Díaz and O. Sigmund, 'Checkerboard patterns in layout optimization', Struct. Optim., 10, 40-45 (1995).

27. M. P. Bendsøe, Optimization of Structural Topology, Shape and Material, Springer, Berlin, 1995. 